\title{
Concept of a Pitot Tube Able to Detect Blockage by Ice, Volcanic Ash, Sand and Insects, and to Clear the Tube
}

\author{
David A. JACKSON \\ School of Physical Sciences, Applied Optics Groups, University of Kent, UK \\ ${ }^{*}$ Corresponding author: David A. JACKSON_Ｅ-mail: d.a.jackson@kent.ac.uk
}

\begin{abstract}
A conceptual coaxial Pitot tube (PT) has been developed using fiber optic sensors combined with actuators to monitor and maintain its correct operation under different environmental conditions. Experiments were performed showing that the dynamic and static tubes can be cleared of ice. It was also demonstrated that the dynamic tube could be cleared of dust and sand which was not the case for the static tube in the coaxial configuration. An approach was proposed to overcome this problem involving a conventional configuration where the static tube was operated independently orthogonal to the dynamic tube, and a second set of sensors and actuators was used. Sensors and associated actuators were developed for temperature and intensity for a linear PT. The aim of this work is to propose a solution for a problem that has caused the loss of the lives of many passengers and crew of aircraft. Resources were not available to test a full implementation of a PT incorporating the proposed modifications.
\end{abstract}

Keywords: Pitot tube, instrumentation, FBG spectrometer, pressure, temperature, optical intensity

Citation: David A. JACKSON, "Concept of a Pitot Tube Able to Detect Blockage by Ice, Volcanic Ash, Sand and Insects, and to Clear the Tube," Photonic Sensors, 2015, 5(4): 298-303.

\section{Introduction}

The Pitot tube [1] (PT) was introduced in 1732; it still remains a key sensor for all modern aircrafts. The PT can be configured in different formats but the designs are invariably based on two tubes: one of the tubes is aligned with the direction of air velocity, the dynamic pressure tube (DPT), and the other, the static pressure tube (SPT) is aligned orthogonally to the flow or velocity direction. The tubes can be mounted coaxially or separately. The pressure data are converted to air speed. If either tube is blocked by ice [2] for example the air speed reading will be false, resulting in a possible fatal accident. Aircrafts have also crashed due to PT blockage caused by flying through volcanic ash and sand. It has also occurred in hot countries where insects have nested in the dynamic tube and where the PT was covered for maintenance and the covers were not removed $[3,4]$. The motivation for this project is to design a PT that not only detects blockage but is also able to clear it, preventing a crash and saving lives.

In Fig. 1, the basic Pitot tube consists of a DPT pointing directly into a fluid flow, which is brought to rest, but has no outlet to allow the flow to continue; the pressure at the input is the stagnation pressure. The SPT is used to measure the local pressure by a ring of small orifices. $\mathrm{S}$ indicates that the pressure and temperature are measured away from the PT with conventional sensors.

Received: 5 August 2015 / Revised: 20 August 2015

(C) The Author(s) 2015. This article is published with open access at Springerlink.com DOI: $10.1007 / \mathrm{s} 13320-015-0272-\mathrm{x}$

Article type: Regular 


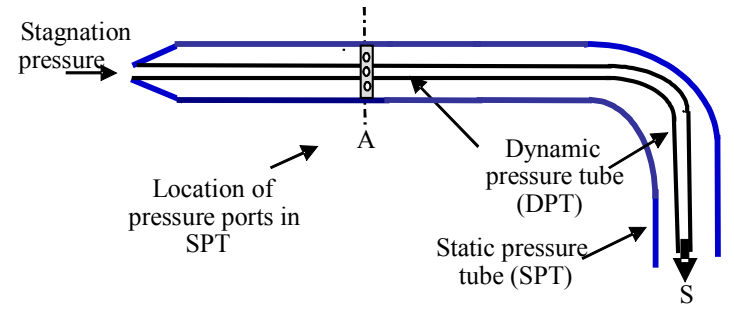

Fig. 1 A conventional coaxial PT, with a central DPT and an SPT with ports to determine ambient pressure.

To measure the airflow, we can use Bernoulli's [5] equation that states: the stagnation pressure $=$ static pressure + dynamic pressure:

$$
P_{1}+1 / 2 \rho \gamma^{2}+\rho g y_{1}=P_{2}+1 / 2 \rho \gamma^{2}+\rho g y_{2}
$$

where $P_{1}$ and $P_{2}$ are the pressures of the DPT and SPT, respectively, $\rho$ is the fluid density, $\gamma$ is the velocity, $y$ is the vertical position from a fixed horizontal plane, and the air flow is assumed to be incompressible. $P_{1}$ and $P_{2}$ are at the same temperature, and the fluid density can be calculated from tables derived using the ideal gas law.

$$
\gamma^{2}=2\left(P_{1}-P_{2}\right) \rho^{-1} \text {. }
$$

\section{Initial configurations considered for optical modifications of the PT}

It was planned to explore two complementary optical approaches to determine if either of the tubes is blocked: (1) a fiber optic topology which can be established directly if either tube is blocked, seen to be the most critical test and (2) a quasi-distributed temperature fiber optic sensor array to control the internal temperature of the Pitot tube at or above a set point via a heater. The heater was used to melt any ice blocking the DPT.

Figure 2 is a schematic diagram of a straight PT simulator incorporating the proposed optical fiber sensors. Both straight and curved PTs are used for aircraft depending on the design and performance of the aircraft, for example a high speed fighter jet will probably have the coaxial PT whereas a passenger plane such as the airbus has the curved PTs. In Fig. 2, a small window allows the laser beam to propagate through the dynamic tube. A serial array of fiber Bragg grating (FBG) temperature sensors is on the bottom of the tube. The laser and detectors are in the processing unit. Initially, we opted to do all tests with the static pressure at atmospheric pressure although the system could operate at other pressures encountered in flight.

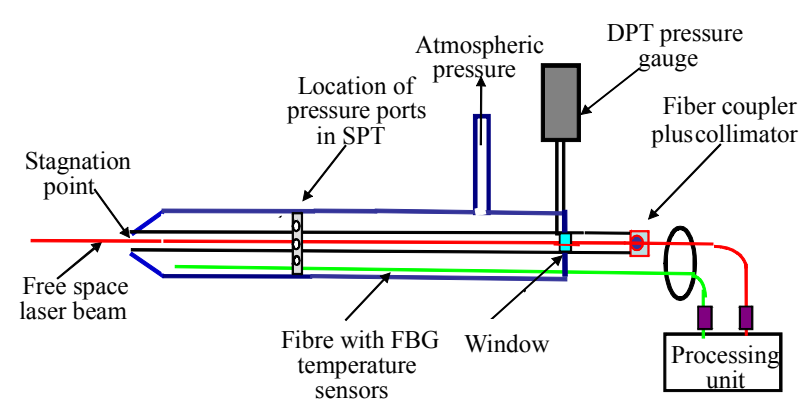

Fig. 2 Straight Pitot tube with optical instrumentation.

Prior to constructing the DPT, a trial was undertaken with a 5-mm inner diameter tube (similar to the DPT planned for the PT) to determine if a "line of sight approach" could offer a simple solution. A smaller diameter laser beam at $1.55 \mathrm{~nm}$ was injected into the tube with the optics correct setup with an unblocked tube; no light was returned to a detector monitoring the back reflected (back scattered) light as expected, indicating the tube was not blocked. When a mirror was aligned normally to the ejected beam and adjusted to send the light back to the detector, a large signal was detected. The overall optical efficiency was close to $100 \%$ allowing for beam splitter losses. Ice was then injected into the output end of the tube where it was anticipated that a significant amount of back scattered light would be detected. Unfortunately, no back scattered light was detected even at input optical powers of up to $20 \mathrm{~mW}$ from a pig-tailed super luminescent diode (SLD). Experiments performed at $800 \mathrm{~nm}$ produced similar results.

\section{Intensity sensor}

An alternative approach for the intensity sensor was adopted, as shown in Fig. 3, where instead of using a "line of sight" approach optical beams are projected across the tube near its distal end. Light 
from the broad band source was coupled into a single mode optical fiber via an isolator which transferred the beam to a Grin lens collimator in contact with a miniature right angle prism that turned the beam by $90^{\circ}$. It was then injected into the tube via a small window mounted on its outside surface and traversed the DPT. The beam was then reflected back across the tube by the combination of a similar window with a small mirror mounted on to the other side of the tube where it was then re-injected into the fiber and transferred to an optical detector. A second beam with an orientation of $90^{\circ}$ to the first beam displaced along the tube $1 \mathrm{~cm}$ further away from the end was generated in a similar manner. The size of the windows was such that there were virtually no effects on the dynamics of the air flow in the DPT. The windows were $0.1 \mathrm{~mm}$ thick by $2 \mathrm{~mm} * 3 \mathrm{~mm}$. When correctly set up, the overall optical efficiency was about $60 \%$. Excellent contrast between a clear DPT and the DPT blocked by ice and sand was demonstrated. The maximum probe operating temperature was $80{ }^{\circ} \mathrm{C}$.

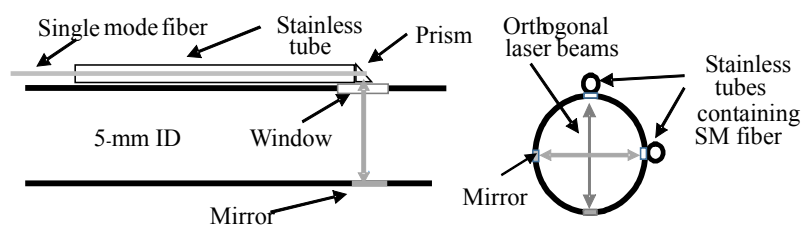

(a)

(b)

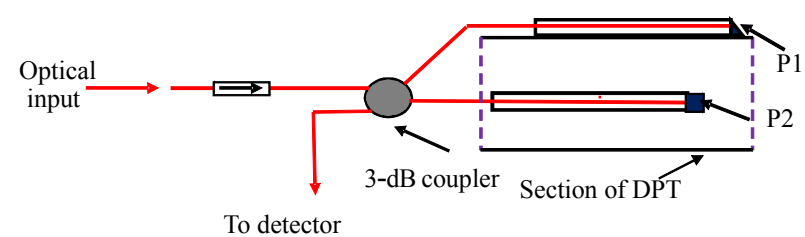

(c)

Fig. 3 Schematic intensity sensor: (a) cross-section of DPT near distal, (b) view into DPT from stagnation point end, and (c) optical arrangement to recover the intensity signal from a DPT [4 fibers are required for a complete PT as shown in Fig. 8, and $\mathrm{P} 1$ and $\mathrm{P} 2$ indicate the prisms in Fig. 3(a)].

\section{Quasi distributed temperature sensor}

Conventional temperature sensors and controllers were used to monitor PTs [6-8] to determine ice accretion and remove it, however only the SPT was heated. Here we heated the DPT directly as it should offer more protection form ice blockage. A fiber optic temperature [9] sensor based on a serial array of $3 \mathrm{FBG}$ temperature sensors with the resolution of about $0.2{ }^{\circ} \mathrm{C}$ at $1545 \mathrm{~nm}, 1550 \mathrm{~nm}$, and $1555 \mathrm{~nm}$ was mounted strain free inside a 0.3 $\mathrm{mm}$ thin wall diameter brass tube located on the bottom of the SPT, respectively. The FBG interrogation system was based on the pigtailed SLD source which when combined with a pigtailed Fabry Perot acted as a low cost tunable source [10] as shown in Fig. 4. A heater made from 100 turns of insulated constantan wire was wrapped around the dynamic tube. The FBG array was spatially deployed such that more weight was given to the FBG close to stagnation point. The output signal from the FBG array controlled the temperature of the SPT via a servo. The temperature of the SPT was continuously monitored as it could rapidly rise in the small volume between the DPT and SPT, for example with 2.2 volts at $0.29 \mathrm{amps}$ applied to the coil, the temperature inside the DPT increased rapidly to $30{ }^{\circ} \mathrm{C}$. To prevent the optical components being damaged by excess heat, the heater was switched off at a specified temperature.

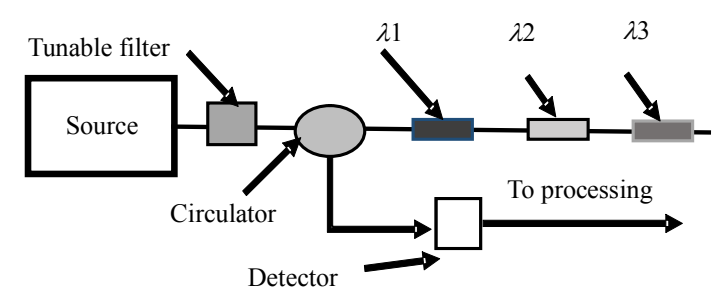

Fig. 4 FBG quasi distributed temperature sensor.

\section{Servo to maintain correct operation of PT}

To test that the ice control servo operated as predicted, the PT was placed in a large polystyrene box which could be set at fixed temperatures in the range of $+5{ }^{\circ} \mathrm{C}$ to $-10{ }^{\circ} \mathrm{C}$ using a simple servo. Figure 5 shows the timing diagram sequence for the 
system operating in the laboratory where during the first time interval T1 the DPT is initially clear and then blocked by ice $\mathrm{T} 2$, then cleared by the heater T3; the outputs from the intensity sensors go to high T3 indicating the DPT is unblocked and T4 and the heater goes off and the intensity sensor goes to high T5. At T6, the intensity sensor goes low but the TC indicates that the DPT is ice free and the heater does not switch on. The tube still remains blocked causing the solenoid valve to open enabling (Fig. 7) high pressure air to sweep the tube clear. The air from the pressure reservoir is maintained at about $20{ }^{\circ} \mathrm{C}$ to minimize condensation on the windows. Other sequences are possible, for instance the valve could be opened every time the intensity sensors indicated tube blockage. The control program was written in Lab View. The close proximity of the SPT and DPT resulted in a very small temperature difference between the tubes such that the heater cleared both tubes from ice nearly simultaneously. Although ice will be cleared from the prototype DPT together with volcanic ash, sand, salt, and insects as discussed above, this is not the case for the SPT in the concentric tube PT configuration because once blocked, the small holes in the outer tube offer too much resistance for the debris to be ejected. A solution proposed here is to configure the PT with two DPTs i.e. DPT1 (dynamic) and DPT2 (static) mounted at a separate location. In the modifications proposed here, the outer tubes are sealed with the FBG arrays mounted on the inside surface, in addition it also protects both the heater and the intensity sensors from any environmental effects. DPT1 is mounted parallel to the flow direction, and DPT2 is mounted orthogonally to the direction of the airflow. Similar sensors and actuators are used to clear both DPTs. The static pressure DPT2 and dynamic pressures DPT1 are measured at the same temperature and substituted into (2) generating the forward velocity.

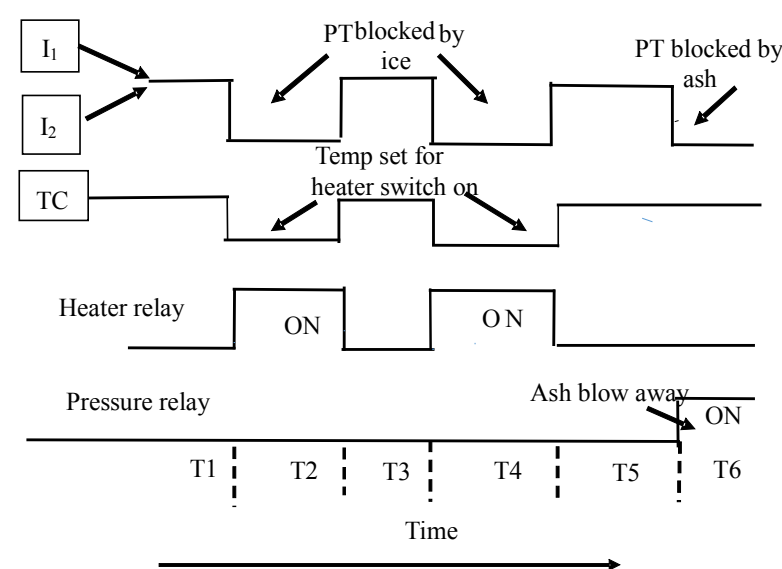

Fig. 5 A time diagram for clearing ice and ash: TC is the temperature control.

\section{Assembled coaxial PT}

The arrows in Fig. 6 identify: 1: DPT; 2: window for intensity sensor; 3: heater; 4: tube containing intensity probe; 5: pressure sensor mount; 6: pressure injection tube; 7: fibers, heater wire.

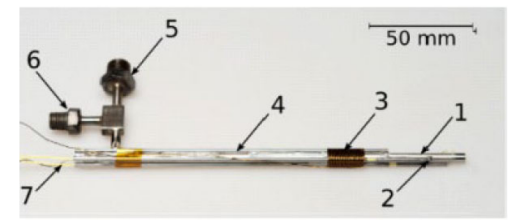

Fig. 6 Instrumented DPT.

The arrows in Fig. 7 identify: 8: SPT; 9: vent to local pressure; 10: pressure sensor; 11: pressure injection port. The dimensions of the DPT are: ID (inner diameter): $5 \mathrm{~mm}$; OD (outer diameter): $6 \mathrm{~mm}$; length: $170 \mathrm{~mm}$, and the dimensions of the SPT are: ID: $12 \mathrm{~mm}$, OD: $14 \mathrm{~mm}$; length: $151 \mathrm{~mm}$. The SICK AG, D-Waldkirch Germany pressure gauge has an operating range of $0-6 \mathrm{bar}$, and the RED DRAGON two way solenoid valve has a pressure range of $0-5.5$ bar.

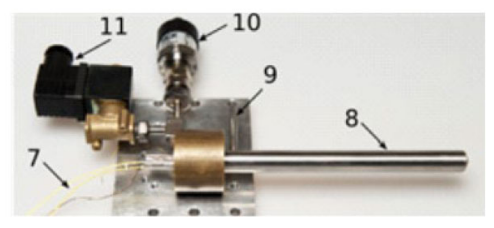

Fig. 7 Completed PT showing pressure gauge and two way solenoid valve on a mount. 


\section{Proposed fully instrumented PT with orthogonal DPT and DPTS interrogated by common instrumentation}

The optical topology in Fig. 8 shows the complete PT with the DPT and the DPTS orthogonally mounted and fully instrumented with the same optical sensors for of each tube, temperature, pressure and optical transmission (electronic controls not shown in Fig. 8). The optical output from the low coherent source with the central wavelength of about $1550 \mathrm{~nm}$ is divided into 2 beams at $3-\mathrm{dB}$ coupler (1), and one of the outputs is coupled into another $3-\mathrm{dB}$ coupler (3) where the output is transferred via the fiber Fabry Perot swept at about $10 \mathrm{kHz}$ to two independent FBG temperature sensors with wavelengths $\lambda 1-\lambda 3$ and $\lambda 4-\lambda 6$ in the DPTs and DPT, respectively. The two back reflected wavelength encoded signals are transferred back to the FBG detectors by 2 circulators enabling the temperatures of each tube to be determined independently. A similar approach is used to recover the optical transmission of each tube. In order to differentiate between the signals, the back reflected signal from each tube is recovered separately as shown in Fig. 3(c). The two independent signals are detected as shown in Fig. 8, and the isolator is used to prevent light being injected to the broad band source as this could cause intensity noise. Each tube is fitted with the same sensors and actuators shown in Figs. 6 and 7. Not all the electrical connections are shown in Fig. 8. The electrical wires connected from system control to the pressure detectors and controllers are not shown.

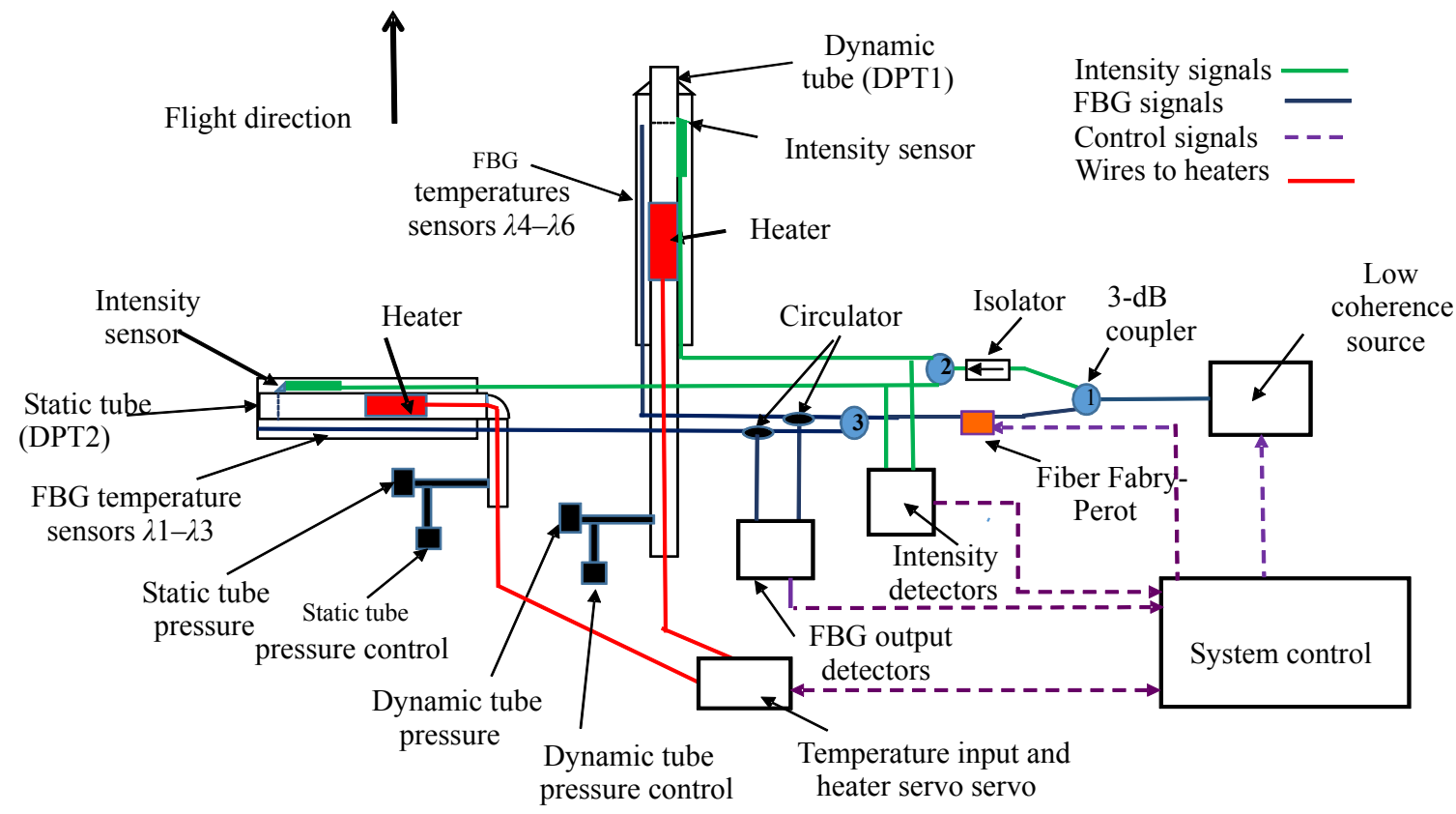

Fig. 8 Proposed fully instrumented PT with orthogonal DPT and SPT interrogated by common instrumentation.

\section{Summary}

The concept of the simulated Pitot tube has been presented in which sensors have been incorporated to detect and remove ice, sand, dust, and any other debris that can block the PT. It is also proposed and has yet to be implemented to use a different but standard configuration for the PT where DPT1 and DPT2 are not coaxially mounted but mounted separately where the same approach to detect ice and debris etc. will be used.

There are two potential problems with the intensity sensor: (1) the debris entering the DPT may not interrupt one of the crossed optical beams; a 
possible solution is to increase the number of crossed beams; (2) the windows may become frosted. Special coatings are available that are wettable and antifogging with frost resistant capabilities offering a solution [11].

Although a pseudo tunable source was used in the initial development of the system, a cheaper and more robust solution would be to use a Bragg grating spectrometer rather that the tunable Fabry-Perot used here, as a commercial unit already has flight approval [12]. The spectrometer replaces the "FBG output detectors" in Fig. 8.

It should be emphasized that the concept of the instrumented PT presented here can be considered as a first step towards greater aircraft safety.

\section{Acknowledgment}

The author acknowledges the contribution of personnel in the Applied Optics Group and the technicians of the University's workshop to the project.

Open Access This article is distributed under the terms of the Creative Commons Attribution License which permits any use, distribution, and reproduction in any medium, provided the original author(s) and source are credited.

\section{References}

[1] G. Brown, "Henry Darcy and the pitot tube," in
International Engineering History and Heritage: Improving Bridges to ASCE's 150th Anniversary, Houston, pp. 360-366, 2001.

[2] G. Traufetter, "Death in the Atlanticthe last four minutes of Air France Flight 447" caused by ice blocked Pitot tube on route from Rio to Paris, DER SPIGEL, 2009.

[3] Wikipedia, "Boeing 757-225 Birgenair flight 301 Pitot tube blocked by wasp nest caused it to crash", 1996.

[4] J. T. McKenna, Blocked Static Ports Eyed in Aeroperu 757 Crash, Aviation Week and Space Technology, pp. 76, 1996.

[5] G. K. Batchelor, An introduction to fluid mechanics. Cambridge, U. K.: Cambridge Mathematical Library, 2000.

[6] Y. Rao, D. J. Webb, D. A. Jackson, L. Zhang, and I. Bennion, "In-fiber Bragg-grating temperature sensor system for medical applications," Journal of Lightwave Technology, 1997, 15(5): 779-785.

[7] C. R. Larkin, "Electrically heated pitot tube," U.S.A Patent 2510986A, Jun. 13, 1950.

[8] P. O. Jarvien, "Aircraft Pitot static tube with ice detection," U.S. Patent 8060344B1, 2011.

[9] J. Debro and T. T. Grolly, "System and method for detecting blocked Pitot-Static Ports," U.S.A Patent 20121123704, 2012.

[10] D. A. Jackson and M. J. Cole, "Fiber optic interrogation systems for hypersonic and low velocity impact studies," Photonic Sensors, 2012, 2(1): 50-59.

[11] H. Lee, M. Alcaraz, M. F. Rubiner, and R. E.Cohen, "Zwitter-wettability and antifogging coatings with frost-resisting capabilities," ACS Nano, 2013, 7(3): 2172-2185.

[12] BaySpec, FBG Interrogation analyzer, BaySpec Inc., California, U.S.A. 